\title{
25-Hydroxy vitamin D levels and endothelial vasodilator function in normotensive women
}

\author{
Sibel Ertek ${ }^{1}$, Ebru Akgül' , Arrigo F. Cicero ${ }^{3}$, Utku Kütük², Selda Demirtaș ${ }^{4}$, Sengül Çehreli², \\ Gürbüz Erdoğan ${ }^{1}$
}

\begin{abstract}
${ }^{1}$ Endocrinology and Metabolic Diseases Department, Ufuk University Medical Faculty, Dr. R. Ege Hospital, Ankara, Turkey

${ }^{2}$ Cardiology Department, Ufuk University Medical Faculty, Dr. R. Ege Hospital, Ankara, Turkey

${ }^{3}$ Internal Medicine, Aging and Kidney Diseases Department, Bologna University, Atherosclerosis and Metabolic Diseases Research Center, Bologna, Italy

${ }^{4}$ Biochemistry Department, Ufuk University Medical Faculty, Dr. R. Ege Hospital, Ankara, Turkey
\end{abstract}

Submitted: 15 September 2011

Accepted: 26 October 2011

Arch Med Sci 2012; 8, 1: 47-52

DOI: 10.5114/aoms.2012.27280

Copyright $\odot 2012$ Termedia \& Banach

\begin{abstract}
Introduction: Vitamin D was shown to be related to endothelial function and blood pressure. Reactive hyperaemia index (RHI) measurement by pulse arterial tonometry is a new method to evaluate vasodilator function of endothelium. We aimed to evaluate the relationship between vitamin $\mathrm{D}$ levels and $\mathrm{RHI}$ in women.

Material and methods: We enrolled 56 normotensive, nonsmoker, normolipidemic and normoglycemic women, (23 with $25-\mathrm{OH}$-vitamin D levels $>20 \mu \mathrm{g} / \mathrm{l}$, and 33 with values lower than $20 \mu \mathrm{g} / \mathrm{l})$. The cardiologist who was blind for vitamin $D$ results executed measurements by pulse arterial tonometry. The measurement was performed on the lying patient with pre- and post-occlusion measurements of RHI by digital sensors placed on each index finger, by $5 \mathrm{~min}$ intervals. Pulse amplitudes were recorded, pre-occlusion and post-occlusion ratio was compared by the software of device. Stepwise linear regression and multiple regression analyses were performed to evaluate predictors of endothelial function.

Results: The low vitamin D group had a lower RHI value than the normal vitamin $\mathrm{D}$ group $(p=0.042)$. In regression analysis, positive predictors of $\mathrm{RHI}$ were serum $25-\mathrm{OHD}(\beta=0.401 ; 95 \% \mathrm{Cl} 0.010-0.042, p=0.002)$, serum albumin $(\beta=0.315 ; 95 \% \mathrm{Cl} 0.286-2.350, p=0.013)$, and, inversely, serum calcium $(\beta=-0.247 ; 95 \% \mathrm{Cl}(-1.347)-(-0.010), p=0.047)$.
\end{abstract}

Conclusions: Serum 25 -hydroxy vitamin D was significantly related to endothelial functions measured as $\mathrm{RHI}$, even in healthy non-smoker women.

Key words: vitamin D, tonometry, endothelium, endothelial function, reactive hyperaemia index.

\section{Introduction}

Recently, a large body of evidence has highlighted the role of vitamin D in different physiopathological settings other than bone and calcium, including endothelial functions and blood pressure [1, 2]. In fact, cross-sectional analyses and prospective studies revealed that 25 -hydroxy vitamin D (25-OHD) serum levels were inversely related to high cardiovascular risk [3-5] and calcium metabolism and the cardiovascular system may have an

\author{
Corresponding author: \\ Sibel Ertek MD \\ Department of Endocrinology \\ and Metabolic Diseases \\ Ufuk University \\ Medical Faculty \\ Dr. R. Ege Hospital \\ Mevlana Bulvarı (Konya Yolu) \\ No. 86-88 Balgat 06520 \\ Ankara, Turkey \\ Phone: +903122044000 \\ Fax: +90 3122044055 \\ E-mail: sibelertek@yahoo.it
}


interaction [6]. On the other hand, normalisation of 25-OHD level by exogenous supplementation is associated with a slight but significant improvement in blood pressure [7-10].

Beyond all this evidence, the final explanation of the vitamin $D$ antihypertensive effect is yet not fully understood [1]. Since vitamin D receptors are expressed in many tissues, it may have an effect on inflammation, cell proliferation and differentiation [11], all factors that could influence vascular function and health. Preclinical studies and clinical reports showed an inverse association of vitamin $D$ and endothelial function $[12,13]$. Very recently, Harris et al. observed in a small group of African-American overweight patients that vitamin $D_{3}$ supplementation may improve flow-mediated dilatation when compared to placebo [14].

The relationship between vitamin $\mathrm{D}$ and endothelial dysfunction is still not clear. Studies with non-invasive methods to measure endothelial function generally use FMD (flow-mediated dilatation) of large arteries, asymmetric dimethyl arginine measurements or augmentation index calculations from pulse wave velocity $[12,15,16]$.

Reactive hyperaemia index (RHI) measurements taken with a pulse amplitude tonometry (PAT) device were found to be correlated with adverse cardiac events in different studies [17, 18], but there has not been any study examining the relationship between vitamin D levels and RHI measurements with this tonometric method.

In this context, the aim of our study was to evaluate the relationship between endothelial function and vitamin D serum level in a cohort of normotensive, non-smoker, normolipidaemic and normoglycaemic women, evaluated by the tonometric measurement of the $\mathrm{RHI}$.

\section{Material and methods}

\section{Patients}

For this study, we consecutively enrolled 56 normotensive, non-smoker, normolipidaemic and normoglycaemic women, 23 with 25-OHD levels $>20 \mu \mathrm{g} / \mathrm{l}$ (normal range) and 33 with values lower than $20 \mu \mathrm{g} / \mathrm{l}$ (low 25-OHD group) [19]. Patients using vitamin or calcium preparations, or affected by known osteoporosis, renal or hepatic disease were excluded from the study.

Anthropometric parameters were recorded and body mass index (BMI) was calculated as weight in kilograms divided by square of height in metres $\left(\mathrm{kg} / \mathrm{m}^{2}\right)$. Blood pressure was measured by standard sphygmomanometer and recorded from both arms while the patients were in a sitting position. According to the current guidelines [20] the measurements were repeated 3 times with minimum 5-min intervals and the mean value was recorded.
Informed consent was obtained from each patient, and the study protocol conforms to the ethical guidelines of the 1975 Declaration of Helsinki as reflected in a priori approval by the university's Human Research Committee.

\section{Endothelial function measurement}

Pulse amplitude tonometry (PAT) is a technique for non-invasive endothelial function assessment from changes in vascular tone using plethysmographic bio-sensors placed on the fingertips. [21]. It is based on non-invasive peripheral arterial tone signal technology. Standard 5-min occlusion of the brachial artery by a cuff creates a downstream hyperaemic response and changes in arterial tone are measured and the index is calculated automatically by the software of the device. A RHI score of 1.67 and below correlates with endothelial dysfunction [22].

In our study, digital pulse amplitude was measured by Endo-PAT 2000 ${ }^{\text {TM }}$ (Itamar, Endothelial Function Assessment Device, Caeserea, Israel) used with the EndoPAT software system. A cardiologist who was blind to vitamin $D$ results made the measurements. The test took nearly 15-20 min and was performed in a calm and quiet room at $22^{\circ} \mathrm{C}$ temperature, at rest. Each patient received an explanation about the procedure before the test and gave informed consent for the study. The measurement was performed on the lying patient with pre- and post-occlusion measurements of RHI by digital sensors placed on each index finger, at 5-min intervals. Inflation pressure of the device was set $10 \mathrm{mmHg}$ lower than diastolic pressure or $70 \mathrm{mmHg}$ as maximum. Pre-occlusion baseline measurements were made from each fingertip for $2 \mathrm{~min}$ and $10 \mathrm{~s}$. Occlusion measurements were performed by the help of a cuff placed on the forearm with $200 \mathrm{mmHg}$ or $60 \mathrm{mmHg}$ higher than the systolic blood pressure of the patient. Pulse amplitudes were recorded, and pre-occlusion and post-occlusion ratios were compared by the software of the device, in an operator or interpreter independent way. Average pulse amplitudes for each $30 \mathrm{~s}$ were provided by the device software. A RHI and heart rate variability measurements were noted for each patient.

\section{Biochemical test}

Venous blood samples were obtained early in the morning after 12-h fasting. The following parameters were evaluated: fasting blood glucose, total cholesterol, high-density lipoprotein (HDL) cholesterol, low-density lipoprotein (LDL) cholesterol, triglyceride, calcium, phosphorus, ionized calcium, and parathyroid hormone (PTH) levels. Parathyroid hormone levels were measured by electrochemiluminescence immunoassay (ECLIA) by an Elecsys 
2010 analyser with inter-assay coefficient of variation below or equal to $6.5 \%$. Thyroid-stimulating hormone (TSH) was measured by electrochemiluminescence immunoassay (ECLIA) using Immulite 2000 (Diagnostic Products Corp, Los Angeles, CA, USA) and Abbot Architect 2000. Fasting blood glucose, total cholesterol, HDL cholesterol, LDL cholesterol, triglyceride, calcium, phosphorus, and ionized calcium measurements were made using an automatic colorimetric method by Cobas Integra 400 Analyzer. 25-OHD measurements were made using high performance liquid chromatography (HPLC). ALT and AST were measured by automatic colorimetric assay by Cobas Integra 400 Analyzer. All measurements were standardized and performed in central laboratories of Ufuk University Medical Faculty Biochemistry Department.

\section{Statistical analysis}

A specific database was created containing all the available variables. Full descriptive statistics were calculated for each parameter. Normality dis- tribution of continuous variables was tested by the Kolmogorov-Smirnov test. Comparisons between groups were carried out by the application of the Student $t$-test for unpaired samples or by the Mann-Whitney $U$-test. A stepwise linear regression was performed to evaluate the predictor of endothelial function. Stepwise multiple regression analysis was performed to determine the best predictors of RHI among the studied parameters.

A $p$ level less than 0.05 was considered as statistically significant. All statistics were calculated with the help of the SPSS 15.0 (for MS Windows) statistical software.

\section{Results}

The mean \pm SD of the studied parameters is reported in Table I. Comparing subjects with low or normal serum vitamin D level, we observed that the group with low vitamin $D$ also had significantly lower $\mathrm{RHI}(p=0.042)$.

Although calcium, ionized calcium and/or phosphorus levels did not show any significant differ-

Table I. Distribution of variables among normal and low 250HD groups

\begin{tabular}{|c|c|c|c|c|}
\hline Variables & All patients ${ }^{\dagger}(n=56)$ & Normal 25OHD $(n=23)$ & Low $25-\mathrm{OHD}(n=33)$ & Value of $p$ \\
\hline Age [years] & $41.4 \pm 8.6$ & $43.0 \pm 8.2$ & $40.27 \pm 8.7$ & 0.245 \\
\hline BMI $\left[\mathrm{kg} / \mathrm{m}^{2}\right]$ & $26.0 \pm 4.2$ & $26.4 \pm 4.1$ & $25.7 \pm 4.2$ & 0.562 \\
\hline $\mathrm{SBP}[\mathrm{mmHg}]$ & $118.7 \pm 9.4$ & $119.9 \pm 7.6$ & $117.8 \pm 10.4$ & 0.428 \\
\hline $\mathrm{DBP}[\mathrm{mmHg}]$ & $69.0 \pm 6.9$ & $72.6 \pm 5.9$ & $66.4 \pm 6.5$ & 0.001 \\
\hline$H R$ & $78.79 \pm 9.29$ & $78.83 \pm 9.80$ & $78.76 \pm 9.08$ & 0.979 \\
\hline Glucose [mg/dl] & $91.82 \pm 9.67$ & $93.79 \pm 12.48$ & $90.44 \pm 7.00$ & 0.978 \\
\hline Haemoglobin [g/dl] & $13.13 \pm 0.93$ & $13.03 \pm 0.86$ & $13.20 \pm 0.98$ & 0.510 \\
\hline Creatinine $[\mathrm{mg} / \mathrm{dl}]$ & $0.64 \pm 0.09$ & $0.64 \pm 0.08$ & $0.65 \pm 0.10$ & 0.722 \\
\hline Albumin [g/dl] & $4.42 \pm 0.23$ & $4.39 \pm 0.30$ & $4.45 \pm 0.16$ & 0.390 \\
\hline $\operatorname{ALT}[U / I]$ & $17.52 \pm 9.23$ & $20.26 \pm 11.54$ & $15.61 \pm 6.76$ & 0.063 \\
\hline AST [U/I] & $16.77 \pm 7.05$ & $17.09 \pm 9.85$ & $16.55 \pm 4.28$ & 0.780 \\
\hline $\mathrm{TSH}[\mathrm{mlU} / \mathrm{ml}]$ & $1.69 \pm 1.17$ & $1.84 \pm 0.93$ & $1.59 \pm 1.32$ & 0.450 \\
\hline Total cholesterol [mg/dl] & $193.61 \pm 32.40$ & $196.49 \pm 30.42$ & $191.60 \pm 34.03$ & 0.583 \\
\hline LDL-C [mg/dl] & $116.56 \pm 26.81$ & $118.49 \pm 26.67$ & $115.22 \pm 27.23$ & 0.658 \\
\hline $\mathrm{HDL}-\mathrm{C}[\mathrm{mg} / \mathrm{dl}]$ & $54.34 \pm 12.38$ & $54.25 \pm 15.85$ & $54.41 \pm 9.53$ & 0.962 \\
\hline TG [mg/dl] & $109.24 \pm 17.98$ & $105.53 \pm 18.11$ & $111.83 \pm 17.70$ & 0.200 \\
\hline Calcium [mg/dl] & $9.28 \pm 0.35$ & $9.25 \pm 0.39$ & $9.31 \pm 0.32$ & 0.536 \\
\hline Ionized calcium [mmol/l] & $1.09 \pm 0.03$ & $1.08 \pm 0.035$ & $1.10 \pm 0.036$ & 0.292 \\
\hline Phosphorus [mg/dl] & $3.42 \pm 0.47$ & $3.56 \pm 0.51$ & $3.33 \pm 0.42$ & 0.075 \\
\hline $25 \mathrm{OHD}[\mu \mathrm{g} / \mathrm{l}]$ & $21.32 \pm 14.82$ & $36.25 \pm 11.61$ & $10.91 \pm 3.61$ & 0.001 \\
\hline PTH [pg/ml] & $49.71 \pm 17.80$ & $44.16 \pm 14.64$ & $53.57 \pm 18.97$ & 0.051 \\
\hline $\mathrm{RHI}$ & $2.29 \pm 0.97$ & $2.61 \pm 1.11$ & $2.01 \pm 0.81$ & 0.042 \\
\hline
\end{tabular}

$B M I$ - body mass index, $25 O H D-25-O H$ vitamin D, PTH - parathormone, ALT - alanine transaminase, AST - aspartate transaminase, TSH - thyroid stimulating hormone, $T G$ - triglycerides, $L D L-C$ - low-density lipoprotein cholesterol, HDL-C - high-density lipoprotein cholesterol, SBP-systolic blood pressure, DBP-diastolic blood pressure, $R H I$ - reactive hyperaemia index, $H R$ - heart rate, tall parameters are given as mean \pm standard deviation 
ence, there was a difference in PTH levels between groups but it was not statistically significant. Diastolic blood pressure and RHI were significantly lower in the low 25-OHD group than in subjects with normal 25-OHD levels.

The stepwise multiple regression showed that the best $\mathrm{RHI}$ positive predictors were serum $25-\mathrm{OHD}$ ( $\beta=0.026 ; 95 \% \mathrm{Cl} 0.010-0.042, p=0.002)$ and serum albumin $(\beta=1.318 ; 95 \% \mathrm{Cl} 0.286-2.350$, $p=0.013)$, while the negative one was serum calcium $(\beta=-0.678 ; 95 \% \mathrm{Cl}(-1.347)-(-0.010)$, $p=0.047$ ) (Figure 1).

\section{Discussion}

Since the early 1980 s, the discovery of vitamin $D$ receptors in non-classical sites (pancreas $\beta$ cells, hair follicles, some cancer cells, etc) has opened a new point of view on vitamin $D$ metabolism and its role in the body [23]. Vitamin D and related mechanisms were well studied in chronic renal disease patients but data on normal people started to increase recently. However, beyond some interesting evidence it is not yet clear how vitamin $D$ is related to vascular function. Recently it has been observed that the expression of proinflammatory transcription factor nuclear factor $\kappa \mathrm{B}(\mathrm{NF} \kappa \mathrm{B})$ was found to be greater in vitamin $D$ deficient middle aged and older adults: inhibition of NFKB by oral acetylsalicylic acid improved brachial artery flowmediated dilatation especially in vitamin $D$ deficient patients [2]. On the other hand, chronic active vita-

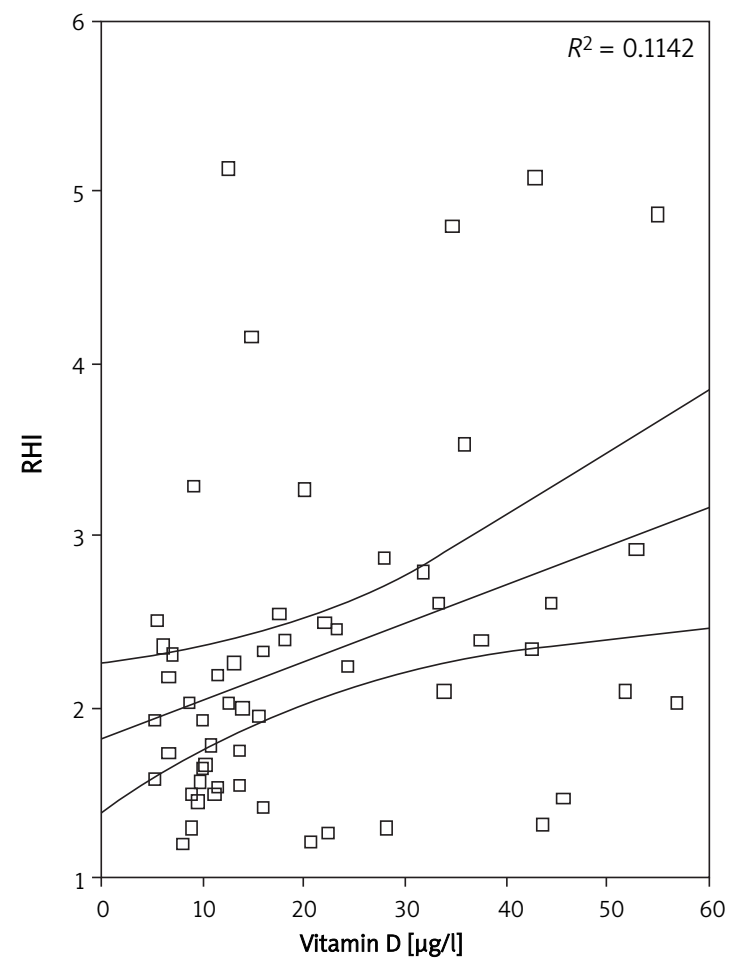

Figure 1. Simple scatter plot of correlation between $\mathrm{RHI}$ and vitamin D levels min D treatment could reduce the levels of reactive oxygen radicals and the expression of cyclooxygenase-1 mRNA, as well [12]. The higher incidence of cardiovascular events in winter could also be partly related to the lower vitamin $D$ levels in this season, since low vitamin $D$ seems to be linked to higher serum lipid peroxidation levels and decreased brachial artery FMD on ultrasonographic measurements [24].

In our study, the $\mathrm{RHI}$ ratio was significantly lower in vitamin D deficient subjects than in subjects with normal serum vitamin $\mathrm{D}$, and $\mathrm{RHI}$ values were strictly predicted by the serum vitamin D levels. Probably the absence of other risk factors such as diabetes, kidney disease, hyperlipidaemia, hypertension or smoking state has a role in RHI measurements higher than endothelial dysfunction thresholds (1.67) even in low vitamin D subjects. The better RHI ratio in normal 25-OHD patients was also associated with a slightly but significantly lower diastolic blood pressure level in this group of normotensive women. Moreover, $\mathrm{RHI}$ was predicted by calcium and albumin separately, but not by ionized calcium levels. The inverse relationship between calcium level and $\mathrm{RHI}$ ratio is not consistent with the previous ex-vivo evidence obtained from a human umbilical vein cord endothelial cell culture which revealed that lower extracellular ionized calcium levels may change endothelial cell functions negatively by decreasing nitric oxide availability and increasing inflammatory mediators [25]. The possible mechanisms of vitamin $D$ action on endothelial functions are not totally elucidated in the medical literature, but the studies reveal that it might be related to the effects of vitamin $D$ on the renin-angiotensin system as providing a "crosstalk" between the heart and kidneys, effects on free radical production, inflammatory and fibrotic processes, carbohydrate tolerance and endothelial progenitor cells [26-29].

Our study has some limitations. First of all, the patient sample was relatively small. Also, PAT measurements evaluate endothelium-dependent vasodilatation as a response to ischaemia, so we cannot suggest a link between 25-OHD levels and endothelium-independent vasodilatation from this study. Meanwhile, since the study groups were all women, it is not possible to extrapolate these results to all people. Another restriction was the lack of comparison between pre- and post-menopausal women, or according to BMI, because of the low patient number. Additionally, the blood pressure measurements were not 24-h ambulatory measurements, which are a more reliable method to evaluate differences in blood pressure level between groups. Furthermore, since we did not include male patients, among whom smoking is relatively common in Turkey, that may affect the PAT 
measurements. On the other hand, the medical literature shows that endothelial results of vitamin $D$ deficiency do not differ between genders $[15,16]$.

However, medical studies examining the effect of vitamin D levels on endothelial dysfunction evaluated by tonometric methods are not common. Pulse amplitude tonometry measurement requires a device and software with digital sensors which are not suitable for multiple use. Therefore this method may have limited use. But the device does have the advantage of using data from both arms and it uses the other arm as a control for systemic vascular changes during measurement. Moreover, previous studies support the use of digital PAT as a measure of peripheral vasomotor function, and nitric oxide has been shown to be an important contributor to augmentation in pulse amplitude measured from the finger tip after ischaemia, supported by a blunted response in PAT in the presence of endothelial nitric oxide synthase inhibitor [30].

In the study of Mheid et al. it was also shown that vitamin $D$ replacement may also improve endothelial function and decreases mean arterial blood pressure in vitamin D insufficiency [31]. Although this may suggest reversibility of endothelial dysfunction in vitamin D insufficiency, some points, such as the effect of the duration of insufficiency, extrapolation of these results to vitamin $D$ deficiency, dose and type of vitamin D preparation to be used, and time to restore normal endothelial function, are still not clear.

Infact, vitamin D may have role in some mechanisms related with endothelial vasodilatation and hypertension, which are not still very clear in medical literature [32].

In conclusion, on the basis of our study, it appears that serum $25-\mathrm{OHD}$ is strongly related to endothelial functions measured from digital pulse amplitude hyperaemic response, even in healthy non-smoker women without hypertension, hyperlipidaemia, or diabetes. Further studies are needed to evaluate the potential importance of vitamin D replacement on prevention or reversal of endothelial dysfunction, and in both genders.

\section{Acknowledgments}

The authors report no conflicts of interest. The authors alone are responsible for the content and writing of the paper.

\section{References}

1. Pilz S, Tomaschiltz A. Role of vitamin D in arterial hypertension. Expert Rev Cardiovasc Ther 2010; 8: 1599-608.

2. Jablonski KL, Chonchol M, Pierce GL, Walker AE, Seals DR. 25-hydroxyvitamin D deficiency is associated with inflammation-related endothelial dysfunction in middleaged and older adults. Hypertension 2011; 57: 63-9.
3. Kendrick J, Targher G, Smits G, Chonchol M. 25-hydroxyvitamin $D$ deficiency is associated with cardiovascular disease in the Third National Health and Nutrition Examination Survey. Atherosclerosis 2009; 205: 255-60.

4. Dobnig H, Pilz S, Sharnagl H, et al. Independent association of low 25 -hydroxyvitamin D and 1,25-dihydroxyvitamin D levels with all-cause and cardiovascular mortality. Arch Intern Med 2008; 168: 1340-9.

5. Stojanovic Ol, Lazovic M, Vuceljic M. Association between atherosclerosis and osteoporosis, the role of vitamin D. Arch Med Sci 2011; 7: 179-88.

6. Yazici S, Yazici M, Korkmaz U, et al. Relationship between blood pressure levels and bone mineral density in postmenopausal Turkish women. Arch Med Sci 2011; 7: 264-70.

7. Burgaz A, Orsini N, Larsson SC, Wolk A. Blood 25-hydroxyvitamin $D$ concentration and hypertension: a metaanalysis. J Hypertens 2011; 29: 636-45.

8. Wu SH, Ho SC, Zhong L. Effects of vitamin D supplementation on blood pressure. South Med J 2010; 103: 729-37.

9. Witham MD, Nadir MA, Struthers AD. Effect of vitamin D on blood pressure: a systemic review and meta-analysis. J Hypertens 2009; 27: 1948-54.

10. Pittas AG, Chung M, Trikalinos T, et al. Systematic review: vitamin D and cardiometabolic outcomes. Ann Intern Med 2010; 152: 307-14.

11. Gouni-Berthold I, Krone W, Berthold HK. Vitamin D and cardiovascular disease. Curr Vasc Pharmacol 2009; 7: 414-22.

12. Wong MS, Delansorne R, Man RY, Svenningsen P, Vanhoutte PM. Chronic treatment with vitamin D lowers arterial blood pressure and reduces endotheliumdependent contractions in the aorta of spontaneously hypertensive rat. Am J Physiol Heart Circ Physiol 2010; 299: H1226-34.

13. Ngo DT, Sverdlov AL, McNeil JJ, Horowitz JD. Does vitamin $\mathrm{D}$ modulate asymmetric dimethylarginine and C-reactive protein concentrations? Am J Med 2010; 123: 335-41.

14. Harris RA, Pedersen-White J, Guo DH, et al. Vitamin D(3) supplementation for 16 weeks improved flow-mediated dilatation in overweight African-American adults. Am $J$ Hypertension 2011; 24: 557-62.

15. Andrade J, Er L, Ignaszewski A, Levin A. Exploration of association of 1,25-OH2D3 with augmentation index, a composite measure of arterial stiffness. Clin J Am Soc Nephrol 2008; 3: 1800-6.

16. Aktoz M, Aktoz T, Tatil E, et al. Asymmetrical dimethyl arginine and severity of erectile dysfunction and their impact on cardiovascular events in patients with acute coronary syndrome. Arch Med Sci 2010; 6: 168-75.

17. Rubinshtein R, Kuvin JT, Soffler M, et al. Assessment of endothelial function by non-invasive peripheral arterial tonometry predicts late cardiovascular adverse events. Eur Heart J 2010; 31: 1142-8.

18. Hamburg NM, Keyes MJ, Larson MG, et al. Cross-sectional relations of digital vascular function to cardiovascular risk factors in Framingham Heart Study. Circulation 2008; 117: 2467-74.

19. Holick F, Binkley NC, Bischoff-Ferrari HA, et al. Evaluation, treatment and prevention of vitamin $D$ deficiency: an Endocrine Society Clinical Practice Guideline. J Clin Endocrinol Metab 2011; 96: 1911-30.

20. Chobanian AV, Bakris GL, Black HR, et al. Seventh report of the Joint National Committee on Prevention, Detection, Evaluation, and Treatment of High Blood Pressure. Hypertension 2003; 42: 1206. 
21. Hamburg NM, Benjamin EJ. Assessment of endothelial function using digital pulse amplitude tonometry. Trends Cardiovascular Med 2009; 19: 6-11.

22. Bonetti PO, Pumper GM, Higano ST, Holmes DR Jr, Kuvin JT, Lerman A. Noninvasive identifications of patients with early coronary atherosclerosis by assessment of digital reactive hyperemia. J Am Coll Cardiol 2004; 44: 2137-41.

23. Norman AW. Minireview: vitamin D receptor: new assignments for an already busy receptor. Endocrinology 2006; 147: 5542-8.

24. Tarcin O, Yavuz DG, Ozben B, et al. Effect of vitamin D deficiency and replacement on endothelial function in asymptomatic patients. J Clin Endocrinol Metab 2009; 94: 4023-30.

25. Talmor-Barkan Y, Rashid G, Weintal I, Green J, Bernheim J, Benchetrit S. Low extracellular Ca2+: a mediator of endothelial inflammation. Nephrol Dial Transplant 2009; 24: 3306-12.

26. Yiu YF, Chan YH, Yiu KH, et al. Vitamin D deficiency is associated with depletion of circulating endothelial progenitor cells and endothelial dysfunction in patients with type 2 diabetes. J Clin Endocrinol Metab 2011; 98 : E830-5.

27. Rostand SG. Vitamin D, blood pressure, and African Americans: toward a unifying hypothesis. Clin J Am Soc Nephrol 2010; 5: 1697-703.

28. Brewer LC, Michos ED, Reis JP. Vitamin D in atherosclerosis, vascular disease and endothelial dysfunction. Curr Drug Targets 2011; 12: 54-60.

29. Cozzolino M, Ketteler M, Zehnder D. The vitamin D system: a crosstalk between the heart and kidney. Eur J Heart Fail 2010; 12: 1031-41.

30. Nohria A, Gerhard-Herman M, Creager MA, Hurley S, Mitra D, Ganz P. Role of nitric oxide in the regulation of digital pulse volume amplitude in humans. J Appl Physiol 2006; 101: 545-8.

31. Al Mheid I, Patel R, Murrow J, et al. Vitamin D status is associated with arterial stiffness and vascular dysfunction in healthy humans. J Am Coll Cardiol 2011; 58: 186-92.

32. Ertek S, Cicero AF, Erdogan G. The relationship between calcium metabolism, insulin-like growth factor-1 and pulse pressure in normotensive, normolipidemic and nondiabetic patients. Arch Med Sci 2011; 7: 776-80. 